\title{
Introducing the idea of entropy to the ontological category shift theory for conceptual change: The case of heat and sound
}

\author{
Alexander Volfson, ${ }^{1}$ Haim Eshach, ${ }^{1}$ and Yuval Ben-Abu ${ }^{2, *}$ \\ ${ }^{1}$ Department of Science and Technology Education, Ben-Gurion University of Negev, \\ P.O.B. 653, Beer-Sheva 8410501, Israel ${ }^{\dagger}$ \\ ${ }^{2}$ Department of Physics and Project Unit, Sapir Academic College, Sderot, Hof Ashkelon 79165, Israel
}

(Received 23 August 2018; published 27 June 2019)

\begin{abstract}
In the present theoretical study, we introduce the entropy concept into Chi's ontological shift theory. Chi distinguishes between two categories of process phenomena, direct and emergent, and claims that incorrectly considering emergent processes as direct ones is one of the sources of students' robust scientific misconceptions. The present study aims to address the needs of high school and undergraduate physics, chemistry, and engineering students being already familiar with the basics of mechanics and the kinetic molecular theory. Acknowledging the contribution of the ontological shift category theory to improving the learning of science, the present paper aims at taking this theory one step further. We argue that more information about scientific phenomena could be gained if we view direct and emergent phenomena as edges of the same scale level of emergency. We show that entropy can be used to evaluate the level of emergency of physical processes. We believe that interpreting scientific phenomena in terms of level of emergency and entropy might promote students' understanding about the underlying mechanisms explaining these phenomena, as well as about the concept of entropy itself. We provide two pedagogical examples of teaching heat and sound using the level of emergency scale and the entropy concept. We demonstrate analytically in these terms: (a) the development of the heat flow rate equation; and (b) the adiabatic nature of the sound propagation process.
\end{abstract}

DOI: 10.1103/PhysRevPhysEducRes.15.010143

\section{INTRODUCTION}

Abstract physics concepts such as sound, heat, electricity, quantum systems, etc., are known to be often misconceived by students as materialistic, direct, and localrealistic phenomena as we explain in the next section. Misconceptions of this type have been reported on a wide range of physics education levels from a middle school up to university students; and known to be stable, robust, and resistant to instruction [1-11]. Therefore, Chi and her colleagues suggest addressing this way of thinking in the teaching process is needed to facilitate the shift towards scientifically correct understanding of these concepts [3-5]. The present article provides a theoretical development of Chi's approach to scientific phenomena analysis and categorization. Ideas suggested in this work intended to offer a more scientifically accurate view of abstract physical concepts or processes, especially those with

\footnotetext{
*Corresponding author. yuvalb@sapir.ac.il

†KeshetCircus@gmail.com
}

Published by the American Physical Society under the terms of the Creative Commons Attribution 4.0 International license. Further distribution of this work must maintain attribution to the author(s) and the published article's title, journal citation, and DOI. nonzero entropy as we show further. These ideas are grounded in psychology of learning and the ontological category shift theory of Chi $[1-5,7,8,12,13]$, and should be further empirically tested in classrooms. Thus, the present work is mainly intended for (a) PER researchers investigating possible causes of students' difficulties regarding abstract physics concepts as well as the ways for providing conceptual change about these concepts, and (b) designers of teaching materials.

In a series of studies, Chi and her colleagues [1-4] developed the ontological shift theory, which shed light on the process of conceptual change among students. They further showed that their theory might also have pedagogical merits, and that if learning environments are designed in such a way that they take their theory into account, conceptual change from materialistic or direct to scientifically correct understanding is achieved more efficiently $[2,4]$. However, Chi's approach offers only a dichotomous view of physics phenomena; i.e., materialistic vs process and or or direct vs emergent. This approach, however, seems to be insufficient for the analysis of real physical phenomena and understanding their inner mechanisms, as we demonstrate below.

Acknowledging the contribution of the ontological shift category theory to improving the learning of science, the present paper aims at taking this theory one step further and 
introduces to it the idea of entropy. Actually, the following two targets should be outlined: (a) developing the dichotomous categorization of physical processes suggested by Chi to a continuous scale that we see to be more scientifically accurate; and (b) providing a newfangled, entropy-based approach with a basic set of tools for physical processes' teaching and analysis. Our approach, as we show further, enables analyzing physical phenomena relating their inner mechanisms by direct entropy evaluation. Such analysis is mostly absent in typical textbooks in thermodynamics and statistical physics, where the authors provide mathematical developments based on entropy differentials usually lacking concrete entropy calculations for real systems (see, for instance, Refs. [14-18]). Maybe, due to this Nobel Prize Laureate, Sommerfeld once wrote,

"Thermodynamics is a funny subject. The first time you go through it, you don't understand it at all. The second time you go through it, you think you understand it, except for one or two small points. The third time you go through it, you know you don't understand it, but by the time you are so used to it, it doesn't bother you anymore" [19] (p. 5).

This cite of Sommerfeld seems to be relevant also for other fields in physics operating abstract concepts such as acoustics, quantum mechanics, etc. We will show that, on the one hand, using entropy contributes to the truthfulness and completeness of physics phenomena description and thus has the potential to facilitate a more accurate and deeper understanding of physics phenomena. On the other hand, the idea of entropy could shed light and further develop Chi's theory itself. In what follows, we (a) introduce the ontological shift approach to conceptual change, (b) discuss the need to introduce the concept of entropy to the ontological shift theory; (c) present the idea of entropy and how it could contribute to the ontological shift theory; and (d) provide pedagogical examples of teaching heat and sound using the concept of entropy.

\section{THE ONTOLOGICAL SHIFT APPROACH}

According to Chi [1,5], students' knowledge is organized in different ontological categories and conceptual change is rooted in categorizing, i.e., the process of identifying or assigning a concept to a category to which it belongs according to a student's views. Once categorized, a concept can "inherit" features and attributes from its category membership that enable a learner to make many inferences and attributions about a novel concept or phenomenon $[4,20]$.

Two relevant ontological categories for this study recognized by Chi are entities and processes. Entities have properties such as "can be contained," "can be pushed," and "has volume"; processes have properties such as "occurring over time." A ball, which belongs to the ontological category of entities, has volume and color, and can be pushed. It is not reasonable, however, to assign the properties of a ball to a basketball game, which belongs to the ontological category of processes. Indeed, a basketball game has no volume or color and cannot be pushed, but it occurs over time. On the other hand, one cannot assign this property to the ball. It is not reasonable to say that a ball occurs over time. From the above, it seems that things belonging to one ontological category may not possess the properties of things belonging to other ontological categories, i.e., ontological categories are mutually exclusive [3].

If how students organize their knowledge is portrayed in this way, serious misconceptions can be viewed according to Chi's approach as a result of incorrect categorization. A concept that has been matched to an incorrect ontological category would inherit incorrectly attributed information, generating serious misconceptions. For instance, students tend to assign many abstract scientific concepts to the entities category instead of the processes category to which they actually belong. Indeed, according to Reiner, Slotta, Chi, and Resnick [12], the initial knowledge that students bring to their studies of science tends to be materialistic or substance based "in the sense that it reflects the novice understands of how material objects and other types of substances behave in the course of everyday life" (p. 1).

Furthermore, Chi distinguishes between two subcategories within the process category, direct (sequential) and emergent processes, claiming that students misconceive some processes of the direct type when they are in fact of the emergent type. In direct processes, each of the system's elements plays a distinct role: the behavior of elements is predefined and predictable, there is an order in the interactions between the elements, and each element causes an identifiable outcome on the global pattern [4].

The process of the wolves' hunt is an example - a kind of an abstract mathematical model-provided by Chi et al. [4] illustrating such a direct process. The process constitutes a series of interactions occurring sequentially in attempting to achieve the goal-get the prey. In this process, each wolf has a certain predefined role: some wolves chase the prey, other wolves distract the prey, and still other wolves block the prey's escape path, followed by the final kill [4]. Although Chi does not use the term "order" to describe the direct process, one may say that direct processes have order. Considering the wolves' hunt process, for instance, one may realize that it is characterized by being an ordered process. Each wolf has a specific role, each wolf's behavior is predictable, each wolf's interaction with the other wolves is predefined and has hierarchy, and there is a clear goal-to catch the prey. The global pattern of the hunt is therefore well ordered.

Emergent processes, on the other hand, require a different kind of causal explanation. They have no identifiable elements that directly cause the displayed or resulting pattern. In such processes, the elements act randomly, 
there is no order of interactions between elements, and a random number of interactions occur simultaneously. As opposed to ordered actions that yield structured patterns, in direct processes, the resulting global pattern in an emergent process is actually a statistical result of the random interactions between the elements, that is, ordered (or disordered) patterns can emerge from a mess.

To illustrate the emergent process, Ref. [4] describes the process of ants searching for sources of food. This process yields an ordered pattern-ants marching in a straight line. Although the result is an ordered structure, the process is not. Here, each element (the ant) does not have any specific predefined role. Rather, each ant operates under a simple program: a random search for sources of food. If food is found, the ant emits pheromones; if an ant smells pheromones, it follows them and emits pheromones of his own; then, the ants carry food to their nest [4]. As opposed to the wolves' hunt prey process, where each element has a specific role contributing to the predefined aim and the global pattern, in the ant searching for food process, each ant has the same role. The interactions between the ants are random, and we have no idea as to which ant will find the food first, which will be the second, and so on. All these random interactions yield a quasistructured pattern-a kind of a straight line. Using the order idea, although there is a seemingly ordered pattern that results from this kind of process, emergent processes have no order. Rather, each element has the same role, the behavior of each element is unpredictable, and random interactions exist between the system's elements.

According to Chi, some robust misconceptions stem from the fact that students lack the collective summing mechanism required for understanding emergent processes. Thus, even if students start viewing a certain phenomenon as a process, it is the wrong process mechanism that they have in mind. For instance, in a series of studies on sound [6,21,22], Eshach showed that students attribute materialistic characteristics to sound, and even if they view sound as a process of propagation from one place to another, they still view this process as a direct one (e.g., as a kind of substance that is being pushed and moved from a sound source to a listener). They do not see sound as an emergent process resulting from random collisions between a medium's particles.

Chi et al. [4] claim that "in order for students to correctly assimilate a process into the appropriate schema, they must be able to recognize which process is a sequential one and which process is an emergent one" (p. 50). They further argue that learning environments considering this need might help students to understand emergent processes and associate emergent processes to the right category. Acknowledging the potential of implementing the idea of direct and emergent processes, we suggest broadening it. In what follows, we explain why and how adding the concept of entropy might improve the idea of emergent processes.

\section{WHY DO WE NEED TO INTRODUCE THE IDEA OF ENTROPY TO CHI'S THEORY?}

According to Chi's theory, as we have said, physical processes can be distinguished as either direct or emergent, i.e., Chi sees direct and emergent processes as dichotomous. We argue that such a dichotomous view is narrow. Consider, for instance, the wolves' hunt process. Just saying that this process is a direct rather than emergent one may hide the difference between the case of, say, three and five wolves. Assume that in the case of three wolves there is one alpha wolf $\alpha$, one chaser $c$, and one distractor $d$; while in the case of five wolves there is one alpha $\alpha$, two chasers $c_{1}$ and $c_{2}$, and two distractors $d_{1}$ and $d_{2}$. One may realize that a difference exists in what might be called the level of directness. While in the case of three wolves, there is only one possible configuration for the hunt process and thus the prediction of each element behavior is accurate, in the case of five wolves, the following are possible configurations: $\left\{\alpha ; c_{1}, c_{2} ; d_{1}, d_{2}\right\},\left\{\alpha ; c_{1}, c_{2} ; d_{2}, d_{1}\right\},\left\{\alpha ; c_{2}, c_{1} ; d_{1}, d_{2}\right\}$, $\left\{\alpha ; c_{2}, c_{1} ; d_{2}\right\}$. The number of possible configurations is 1 ! $\times 2$ ! $\times 2$ !, which indeed yields four options. Since there are more possible configurations for the hunt process to occur, the less we can accurately predict the behavior of each element (wolf), the "less" direct the process is. In other words, we argue that in order to gain a deeper understanding of a phenomenon, it is not enough to attribute it to the direct category, but rather it is worthwhile to consider also the level of directness.

Moreover, we claim that categorizing some phenomena to either direct or emergent processes is not at all possible since under some conditions, a particular phenomenon belonging to a certain concept may be a direct process, while under other conditions, the same phenomenon should be categorized as an emergent process. To illustrate this point, let's refer to heat transfer in air (an ideal gas). According to Chi, heat transfer is an emergent process. Indeed, each air molecule has the same role-move and collide. Molecules that are close to the heat source start moving faster and collide with their neighboring molecules. These molecules also hit their neighbors, and so on. Molecular movements and collisions occur in all directions with the same probability. Heat is transferred in this way from one place to another.

Now, let us look at the extreme case of heat transfer in the air found in a long and narrow horizontal tube (in this case, there is no convection). Assume also that the tube is as narrow as possible and that it is almost one dimensional so the molecules are arranged in a line one after another (see Fig. 1). Assume also that no heat loss or absorption occurs in the system, and that the Brownian motion and thermal fluctuations are neglected.

Imagine, now, that we heat one edge of this tube. As a result, the first molecule oscillates and hits its closest neighbor, and so on. In this special case, the air molecules are restricted to move back and forth only, and there is an 


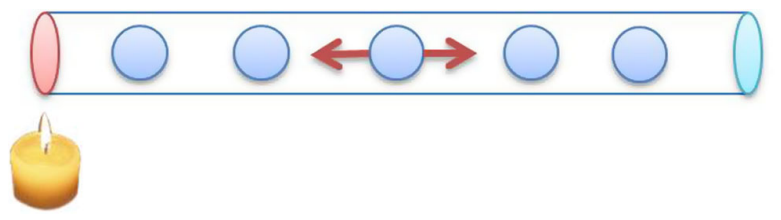

FIG. 1. Heat conduction (schematically) in a one-dimensional hollow tube.

order in the collisions. Therefore, we can predict which molecule will start to move and with which molecule it will collide at every point in time. So, under this condition, heat transfer actually obeys the conditions of a direct, rather than an emergent, process as defined by Chi et al. [4]:

- The elements interact sequentially - the first molecule collides with the second, the second with the third, and so on.

- Elements' interactions are contingent or logically dependent on other agents' interactions - every collision depends on the previous one.

- Agents' interactions terminate when the pattern-level behavior stops-when we stop heating the left edge, the process will terminate.

In summary, as can be seen from the examples above, it is worthwhile considering a kind of emergent or direct level rather than seeing them as dichotomous. In what follows, we will first explain the idea of entropy and then discuss how this idea can be used to quantitate emergency or directness.

\section{THE IDEA OF ENTROPY AND ITS CONNECTION TO LEVEL OF EMERGENCY}

For the purpose of this study, we will use the term entropy as defined by Boltzmann [23]. To understand what entropy is, let's consider a tube full of ideal gas molecules. Because of random collisions between the molecules, there are many possible configurations of the molecules within the vessel. Each configuration at a particular time is called a state of the system. There are many possible arrangements of the gas molecules within each state. For instance, consider the state that all of the molecules are at the bottom right of the vessel. Within this state, there are many possible arrangements of the gas molecules. Entropy of a state relates to the number of possible arrangements of the molecules in this state. The larger the number of possible arrangements in a certain state, the larger is the entropy. A state in which there are more possible arrangements, the more likely it is to find the system in that state. A system's entropy in a certain state of $\Omega$ equiprobable arrangements is defined as $[14-16,23]^{1}$

\footnotetext{
${ }^{1}$ Entropy in thermodynamics books is usually defined as $s=$ $k_{B} \ln (\Omega)$ where, $k_{B}$ is the Boltzmann constant.
}

$$
s=\ln (\Omega)
$$

This definition teaches us that entropy can be related to the amount of information we do not know about the behavior of a system's elements in a certain state [14-16,23]. Indeed, suppose that a system is found in a state with only one possible arrangement. In this case, we know the arrangement exactly and thus the entropy $s=\ln (1)=0$. However, as the number of possible arrangements $\Omega$ within the state grows, i.e., the entropy of the system is larger, the less information we have about the accurate behavior of the system. This is because, in this case, there are many more possibilities for a specific element to have a certain behavior. This is why entropy is also considered a measure of a system's disorder.

By definition, entropy is an additive quantity, i.e., if a system is comprised of $n$ subsystems each of entropy $s_{i}$, the total entropy of the system will be $[14-16,23]$

$$
s=\sum_{i=1}^{n} s_{i}
$$

Let us look again at the three wolves' hunt process and analyze it from the entropy viewpoint. The wolves form a system of three elements: each has a predefined role, as described above. Because each wolf has a predefined role in this system, there is only one way the hunt process can take place, that is, $\Omega=1$ and thus

$$
s_{a}=\ln (1)=0
$$

Now, imagine that there are six wolves in the pack: one alpha, two distracters, and three chasers. There are three subgroups in this system: $\left\{\{\alpha\},\left\{d_{1}, d_{2}\right\},\left\{c_{1}, c_{2}, c_{3}\right\}\right\}$. Since there are two distractors and three chasers, one cannot precisely predict the behavior of each wolf. It can, for instance, be either chaser 1, 2, or 3 that will first catch the prey. Therefore, the more elements in the system that possess the same role, the less we will be able to predict precisely the behavior of each element. This means that although the hunt process can still be characterized as a direct one, we cannot say that the level of directness in the two cases is the same. Using entropy, whereas in the three wolves case it is 1 , in the case of six wolves the entropy is

$s_{b}=\ln (1 ! \times 2 ! \times 3 !)=\ln (1)+\ln (2)+\ln (6)=2.48$,

where, $\Omega=1 \times 2 ! \times 3 !=12$ is the number of equiprobable possible configurations. Therefore, entropy may be used as an indicator of the system's level of directness or emergency. What we suggest here is that a deeper insight into scientific phenomena might be achieved if we consider a kind of "scale of emergency" or what one might call level 
of emergency, and use entropy to this end. In what follows, we will provide pedagogical examples of heat and sound phenomena, and describe how teaching them using the ontological shift theory combined with entropy might improve their understanding.

\section{PEDAGOGICAL EXAMPLE OF TEACHING ABOUT THE HEAT TRANSFER PROCESS USING THE ONTOLOGICAL SHIFT THEORY COMBINED WITH THE CONCEPT OF ENTROPY}

Thermodynamics is considered one of the most difficult domains in physics $[24,25]$. Indeed, many researchers show that learners "...face obstacles in mapping abstract, theoretical understanding of thermodynamic principles" [26] (p. 704). One of the barriers students may face stem from their materialistic thinking, which is defined by Eshach et al. [22] as their tendency to associate materialistic properties to scientific concepts. Indeed, Reiner et al. [12] and Chi [1,3] argue that naïve students tend to assign heat to the entities category, and thus explain phenomena related to heat in terms of matter and the characteristics they attribute to matter. For instance, students mistakenly view heat as being a kind of matter that flows from one place to another, and can be contained and accumulated in a closed space [12]. Such materialistic views of heat were also found among undergraduate students [27]. Furthermore, when students start to assign heat to the processes category, they still tend to perceive heat transfer as a direct process according to which, for instance, "hotness" moves from one area to another area, or "hot molecules" travel from one location to another [1].

To facilitate students' understanding of heat, materialistic thinking should not be ignored by teachers. On the contrary, teachers should be aware of their students' tendency to attribute materialistic properties to heat [1]. We agree with Clement's [28] suggestion that naive materialistic conceptions might provide a good starting point for instruction, and argue that teachers should scaffold their students and lead them from materialistic ideas to scientific ones. Furthermore, we agree with Chi [3] that the direct teaching of direct and emergent processes could help students to overcome some of the barriers they face. Indeed, according to Chi, "because emergent and direct processes are different in kind, with mutually exclusive properties, confrontation needs to reject the misassigned category and build the alternative emergence category, perhaps through direct instruction using contrasting cases" (p. 80).

We agree with Chi $[1,2,4]$ that instruction should "focus on teaching the underlying causal structure of emergent processes via the ontological attributes. The idea is that if we can help students build a general structure or schema of emergence first ..., then presumably learning, in the sense of assimilating and integrating new knowledge with existing knowledge, can be more easily undertaken because the relevant cognitive structure will already have existed." [1] (p. 194). That is, according to Chi students should be taught the general framework of direct and emergent analysis on the first stage, applying simple examples such as the wolves' hunt. And only later, emergent physical phenomena-heat transfer in this case-should be introduced using the existing schema and being analyzed by the tools learned on the first stage.

However, focusing only on whether the heat transfer process is direct or emergent per se is not enough. We argue that analyzing and interpreting heat transfer in terms of entropy might provide an even deeper insight into scientific concepts. To illustrate this point, we provide the example of heat conduction. Usually undergraduate textbooks introduce learners with the following formula of heat flow rate in a metal rod:

$$
\begin{gathered}
H=\frac{k\left(T_{H}-T_{C}\right)}{L} A \\
\Downarrow \\
H \propto A,
\end{gathered}
$$

where $H$ is the amount of thermal energy transferred per unit of time through the rod, $T_{H}>T_{C}$ are the temperatures of the hot and cold edges, respectively, $L$ is the length of the rod, $k$ is the thermal conductivity factor of the metal, and $A$ is the cross section. Halliday and Resnick [29] explain that "the vibration amplitudes of the atoms and electrons in the [metal rod] become relatively large because of the high temperature applied. These increased vibrational amplitudes, and thus the associated energy, are passed along the [rod], from atom to atom, during collisions between adjacent atoms. In this way, a region of rising temperature extends itself along the [rod]." (p. 534). The textbooks further explain that this equation is obtained experimentally and some exercises are given (see, for instance, Refs. [29,30]). However, there is usually a lack of a more detailed explanation regarding the inner mechanisms of the process of heat conduction as well as some analytical development of proportion [Eq. (4b)]. While we believe that questions like what are the atoms' or electrons' vibration directions and why is heat flow rate proportional precisely to the cross section and not to cross section squared should be addressed, we believe that the idea of emergency level defined by entropy could provide students with a deeper insight into the process and explain more the physical meaning of Eq. (4b).

First, we suggest explaining that heat conduction through a horizontal tube with different cross-sections may be characterized by different levels of emergency. As we previously explained, in a very thin almost onedimensional tube, heat conduction is a direct process, and as its cross-section increases, the process becomes more 
emergent as there are more options for collisions between the gas molecules. Also, as was argued, the level of emergency can be determined by assessing the system's entropy. Let us now show that using the concept of entropy, we can provide a more in-depth explanation of Eq. (4a).

Suppose that for a given gas temperature, the molecules inside the tube have $\Omega_{\mathrm{sp}}$ possible spatial arrangements and $\Omega_{\mathrm{vel}}$ accessible distributions of velocities. Therefore, the number of equiprobable configurations of the molecules in the system is $\Omega=\Omega_{\mathrm{sp}} \Omega_{\mathrm{vel}}$ and thus the system's entropy is [15]

$$
s=\ln \left(\Omega_{\mathrm{sp}}\right)+\ln \left(\Omega_{\mathrm{vel}}\right) .
$$

Suppose now we add a small amount of heat $Q$ to the gas by heating the left edge of the tube. ${ }^{2}$ This adds some kinetic energy to the molecules, and thus $\Omega_{\mathrm{vel}}$ increases by $\Omega_{\mathrm{vel}}^{Q}$, yielding an increase of the system's entropy by

$$
\Delta s_{Q}=\ln \left(\Omega_{\mathrm{vel}}^{Q}\right) .
$$

This entropy extension points to the emergent nature of every heating process.

As is known from the literature, the change of heat and entropy in an isothermal process, i.e., for the addition of a small amount of heat, $d Q$ can be described by the following formula:

$$
d s=\frac{d Q}{k_{B} T} \Rightarrow d Q=k_{B} T d s,
$$

where $k_{B}=1.38 \times 10^{-23} \frac{\mathrm{Joule}}{\mathrm{K}}$ is the Boltzmann constant (one can find this formula's development in Refs. [15,16,29]). Accordingly, heating one edge of the tube leads to an increase in the entropy of the gas in the tube.

Now let us return to the process of heat flow through the tube. Heat flow rate in general is defined as follows: ${ }^{3}$

$$
H=-\frac{d Q}{d t}
$$

Substituting Eq. (6) into Eq. (7), we get

$$
H=k_{B} T \frac{d s}{d t},
$$

which means that heat flow can be expressed in terms of entropy flow. To reach Eq. (4b), let us consider again the long hollow tube of cross-section $A$ filled with an ideal gas of density $\rho$. There are $n$ molecules found in a length element $d x$. Each molecule can oscillate and collide with its

\footnotetext{
${ }^{2}$ In this case, its temperature stays almost constant and the process is called isothermal.

${ }^{3}$ The minus represents the fact that heat flows from the hot to the cold edge.
}

$m$ closest neighbors. We assume these collisions to be equiprobable. Adding some energy $d Q_{1}$ to a certain molecule causes it to oscillate and collide, and thus the entropy increases by $d S_{1}=\ln (m)$. There are $n=\rho A d x$ molecules in this length element. Thus, adding heat $d Q$ to the length element increases its entropy by

$$
d s=\rho A \ln (m) d x .
$$

We divide Eq. (9) by $d t$ and get the entropy flow rate proportional to the tube's cross section $A$ :

$$
\frac{d s}{d t}=A \rho \ln (m) \frac{d x}{d t} .
$$

To finally reach Eq. (4b), we must substitute Eq. (10) into Eq. (8):

$$
H=k_{B} T \frac{d s}{d t}=A k_{B} T \rho \ln (m) \frac{d x}{d t} \Rightarrow H \propto A .
$$

In summation, we show that using the idea of entropy may help us explain the mechanism of the heat conduction process. We explained that heating one edge of the tube increases the molecules' velocities. This increases the number of possible configurations of molecules, resulting in higher entropy, which is an indicator of the level of emergency. Indeed, as more molecules are found in the tube's cross section, more collisions will probably occur at each point in time, causing the entire process to be more emergent and the heat flow rate to increase (see Fig. 2) and
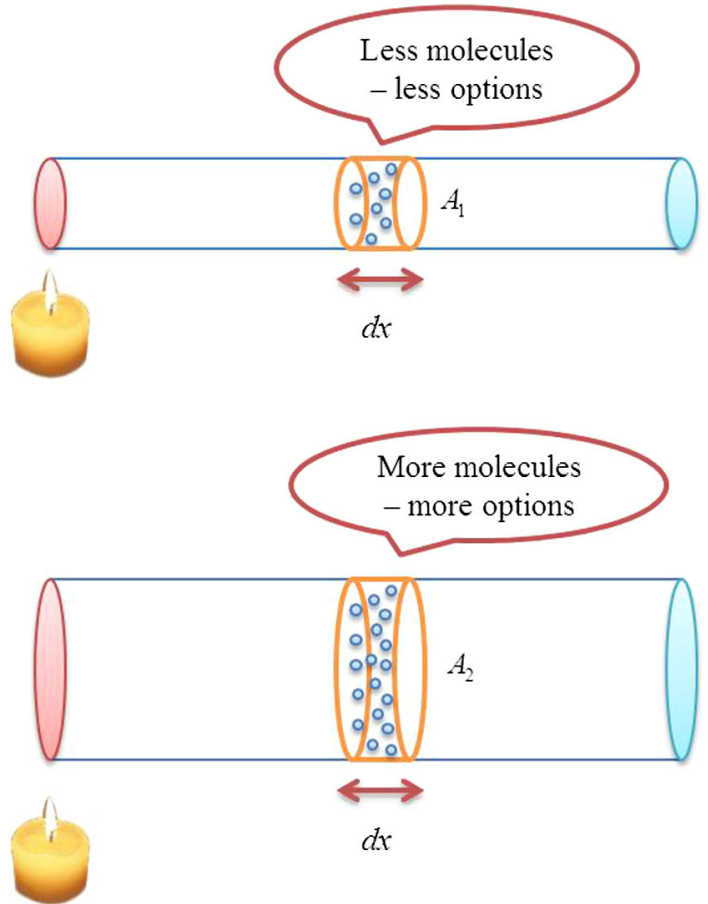

FIG. 2. The larger the cross section, the more collisions are probable. 
vice versa. As the tube becomes narrower, the entropy flow through the tube decreases. This is to say, the process becomes less emergent. Until at the limit of almost a onedimensional tube $\left.\frac{d s}{d t}\right|_{A \rightarrow 0}=0$ and the process becomes almost direct as we have shown in Sec. III. This explanation is also relevant for heat conduction in metals: the heat transfer process in metals is mainly conducted by valence electrons, which can be considered to be an ideal gas in good approximation (see, for instance, Ref. [31]).

\section{SOUND PROPAGATION}

Examining the criteria by Chi et al. [4] for emergent processes, one can realize that sound, like heat, is an emergent process. This is because of the following:

- All gas molecules interact in the same uniform way by following the same set of rules-oscillations and collisions correspond to the wave equation.

- All agents interact independently of one another-a collision of every two molecules is independent of the other collisions.

- The interactions of the entire collection of gas molecules together "cause" the observable patterncompression and rarefaction zones in the sound wave.

However, according to physics textbooks (see, for instance, Ref. [14]), sound propagation as opposed to heat transfer is an adiabatic process. An ideal adiabatic process from the thermodynamic point of view is a process that “... occurs so rapidly or occurs in a system that is so well insulated that no transfer of energy as heat occurs between the system and its environment" [29] (p. 532). Such processes are characterized by low entropy [14]. Eq. (6) demonstrates this fact algebraically.

The same can be argued also from the information point of view. Indeed, while in the case of heat transfer, gas molecules move and collide in random directions, molecules affected by a sound wave mostly oscillate back and forth. That is, more information is known about the molecules' behavior in the case of sound propagation and thus its entropy is much smaller. This means that a substantial difference exists between heat and sound. The dichotomous distinguishing emergent-direct process is not enough in this case as it actually hides this difference. However, examining the levels of emergency can explain this gap.

Moreover, applying entropy enables making a comparative analysis of physical phenomena, for instance, a comparison of heat transfer vs sound pulse propagation in a hollow tube filled with an ideal gas (air) when the amounts of energy added to the system by heating and the sound pulse are equal. Such a comparison has the potential of facilitating a better understanding of both phenomena. Indeed, drawing comparisons help to perceive, comprehend, and reason about relations. It is called relational reasoning in the literature and may be defined as the ability to consider relationships between multiple elements (objects of attention), which is directly linked to the capacity to think logically and solve problems in novel situations [32]. Relational reasoning allows learners to derive symbolic, abstract, and conceptual knowledge representations that are generative in that children and adults can then use them broadly in new contexts to reason about new elements [32-35]. Therefore, such a comparative analysis of processes is expected to enable their better understanding.

To do so, let us examine the sound propagation process in the same long hollow tube filled with air (ideal gas) as we did previously for the heat transfer process analysis. The behavior of each air molecule is random since the sound propagation mechanism in air is similar to that of heat transfer. So, for instance, one cannot predict whether molecule $i$ will collide with molecule $j$ or molecule $k$ at a certain time $t$. This fact demonstrates the emergent nature of the sound propagation process. However, this process is much less emergent relative to the heat transfer one. Indeed, for the same amount of energy $Q$ added to the system by heating [see Eq. (5b)] and by a sound pulse, the heat transfer process adds more entropy to the system than the sound pulse. This is because heating causes the medium molecules to oscillate in random directions while all the directions are equiprobable, whereas molecules excited by a sound pulse have more and less probable oscillation directions. In this case, we have additional information about the behaviors of the elements. Thus, although both processes are emergent, they differ by level of emergency.

To analyze situations with preferred behaviors of elements whereby certain configurations of elements have a higher probability to occur, we should start again from the wolves' hunt example. Let us first suppose that there are three wolves in the pack, three roles are accessible (alpha, chaser, and distracter) and all the roles' distributions $(\{\alpha, c, d\}$, $\{\alpha, d, c\},\{c, \alpha, d\},\{d, \alpha, c\},\{c, d, \alpha\},\{d, c, \alpha\})$ are equiprobable. In this case, the entropy of the hunt process will be simply $s_{c}=\ln (3 !)=\ln (6)=1.79$.

However, imagine now another situation when Wolf 1 is the strongest wolf in the pack, therefore his probability to be the alpha is almost one. Wolf 2 usually runs faster than Wolf 3 , so we suppose in this example that the probability of Wolf 2 to be the chaser and Wolf 3 the distracter is $p_{1}=0.8$. However, another distribution of roles is also possible, that is, Wolf 1 is the alpha, Wolf 2 is the distracter, and Wolf 3 is the chaser. The probability of the last distribution is $p_{2}=0.2$ (we assume the two configurations to be independent). This situation is in a sense parallel to the case of air molecules found in a certain cross section of a tube excited by a sound wave. Indeed, each molecule can move mostly back and forth-two configurations of velocities, whereas the probability of these directions is a function of time. When, for instance, a sound wave exerts forward pressure on the cross section, the forward direction is much more probable, and vice versa. The probabilities of 
all other oscillation directions are much smaller. Calculating the entropy in such cases of nonequiprobable configurations or behaviors of a system requires using the Shannon formula [36]:

$$
\begin{aligned}
s & =-\left[p_{1} \ln \left(p_{1}\right)+p_{2} \ln \left(p_{2}\right)+p_{3} \ln \left(p_{3}\right)+\cdots\right] \\
& =-\sum_{i=1}^{\Omega} p_{i} \ln \left(p_{i}\right),
\end{aligned}
$$

where $p_{i}$ is the probability for the $i$ th configuration. Eq. (12a) may be rephrased as follows:

$$
\begin{aligned}
s & =p_{1} \ln \left(\frac{1}{p_{1}}\right)+p_{2} \ln \left(\frac{1}{p_{2}}\right)+p_{3} \ln \left(\frac{1}{p_{3}}\right)+\cdots \\
& =\sum_{i=1}^{\Omega} p_{i} \ln \left(\frac{1}{p_{i}}\right) .
\end{aligned}
$$

As to the wolves, the entropy in such a case will be

$$
s_{d}=0.8 \ln \left(\frac{1}{0.8}\right)+0.2 \ln \left(\frac{1}{0.2}\right)=0.5
$$

Although both wolf hunt processes-equiprobable roles' distribution and nonequiprobable roles-for the same number of wolves are emergent, they differ in the amount of information we know about the behavior of each wolf and thus by the level of emergency and entropy. Indeed, the first case yielded entropy of 1.79 while the second one yielded 0.5. Examining Eqs. (12a) and (12b) one may realize that the entropy of a state with multiprobable arrangements, i.e., $p_{i}>p_{j}$, is smaller than the entropy of a state when all the arrangements are equally probable to take place. That is,

$$
s_{c}\left(p_{i}=p_{j}\right)>s_{d}\left(p_{i}>p_{j}\right), \quad i \neq j .
$$

The same can be expected for the heat transfer and sound propagation processes. Indeed, "heated" air molecules are free to move in all directions with the same probability, while molecules excited by a sound pulse (wave) have more and less probable directions of motion at every moment of time so their behavior can be predicted by the wave function with a high degree of probability.

Let us demonstrate this analytically. Like in the case of heat, we consider the same hollow tube of cross section $A$ filled with an ideal gas of density $\rho$. There are $n$ molecules found in a length element $d x$. Suppose that a sound pulse of the same energy $Q$ as in the case of heat propagates along the tube and at a certain moment it pushes all the $n$ molecules found in $d x$ forward (see Fig. 3). As we showed in the case of heat, the energy of the pulse increases the number of distributions of velocities by $\Omega_{\mathrm{vel}}^{S}$ (upper index $S$ stands for sound) when $\Omega_{\mathrm{vel}}^{S}=\Omega_{\mathrm{vel}}^{Q}$. However, the

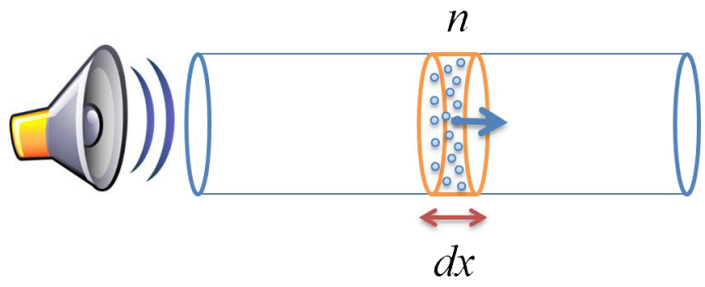

FIG. 3. The element of length $d x$ is pushed forward. The arrow shows the most probable direction of the $n$ molecules' motion.

distributions of velocities are not equiprobable. The $\Omega_{+\hat{x}}$ distributions where the molecules' velocities are of a component in the positive $x$ axis direction are much more probable than all the other distributions. For further simplicity, we suppose that all the $\Omega_{+\hat{x}}$ distributions are of the same probability $p$, whereas $q=1-p$ is the probability of all the other distributions, and, as it was said, $p>q$.

According to Eq. (12b), the entropy added to the $n$ gas molecules by the sound pulse is

$$
\Delta s_{\text {sound }}=n\left[p \ln \left(\frac{1}{p}\right)+q \ln \left(\frac{1}{q}\right)\right]
$$

Consequently with Eq. (14), the entropy of sound is smaller than the entropy of heat transfer [Eq. (5b)]

$$
\begin{aligned}
n\left[p \ln \left(\frac{1}{p}\right)+q \ln \left(\frac{1}{q}\right)\right]<n \ln \left(\Omega_{\mathrm{vel}}^{Q}\right) \\
\Delta s_{\text {sound }}<\Delta s_{Q} .
\end{aligned}
$$

This demonstrates analytically that although sound propagation is an emergent process, its level of emergency is lower than the level of emergency of heat transfer.

Now let us go one step further and demonstrate using entropy the fact that sound can be treated as an adiabatic phenomenon. ${ }^{4}$ For a relatively strong acoustic pulse pushing the cross section in the $+\hat{x}$ direction at a certain moment, it is obvious to expect that most of the molecules will move forward so that $q \rightarrow 0$. That is, the probability of any other motion except in the $+\hat{x}$ direction is very small, as it happens for real sounds [29]. In this case, one should calculate the following limit (using the L'Hôpital's rule):

\footnotetext{
${ }^{4}$ Typical textbooks introduce sound as an adiabatic process almost by definition, see Refs. [14,29], and others. Raichel [37], for instance, argues that "The transmission of the sound through the fluid [air] results in low values of spatial temperature gradients at audio frequencies, resulting in almost no heat transfer between warmer and cooler regions of the plane wave. Thus the ongoing thermodynamics process may be considered an adiabatic process" (p. 26).
} 


$$
\lim _{q \rightarrow 0} q \ln \left(\frac{1}{q}\right)=\lim _{q \rightarrow 0} \frac{\ln (1 / q)}{1 / q}=\lim _{q \rightarrow 0} \frac{q_{i}\left(-1 / q^{2}\right)}{\left(-1 / q^{2}\right)}=\lim _{q \rightarrow 0} q=0 .
$$

Leaving the entropy of sound [by Eq. (15)] to be

$$
\Delta s_{\text {sound }}=n p \ln \left(\frac{1}{p}\right) .
$$

Now, the less is $q$ and the more $p \rightarrow 1$, the less is the entropy brought by the sound pulse to the system. Indeed,

$$
\lim _{p \rightarrow 1} n p \ln \left(\frac{1}{p}\right)=0
$$

thus making $\Delta s_{\text {sound }}$ to be very small and characterizing the whole process of sound propagation as being adiabatic [14]. It is worth mentioning here that considering the sound propagation process as adiabatic is crucially important for its understanding. For instance, considering the adiabatic nature of sound enabled Laplace at the beginning of the 17th century to develop the correct equation for sound velocity in a gas and precisely get the true value of sound velocity analytically in air $[38,39]$.

\section{DISCUSSION AND CONCLUSIONS}

Recognizing the merits of the ontology shift theory to explain conceptual change processes and its pedagogical potential to promote students' learning [2,4], the present paper suggested broadening it, and introduced to it the idea of entropy. Ideas suggested in this work are aimed to suggest a more scientifically accurate view of abstract physical concepts or processes, especially those with nonzero entropy such as heat transfer, sound propagation, etc. These ideas are grounded in the psychology of learning and the ontological category shift theory of Chi $[1-5,7,8$, $12,13]$. It is time now for these ideas to be empirically examined. That is, conceptual change provided by teaching materials based on the proposed approach should be measured and compared to the one provided by classical materials and materials based on the "original" approach of Chi per se. Also, the proposed entropy-based approach can be applied by PER investigators to identify and analyze possible students' misconceptions regarding abstract physics concepts as well as further developing of ways for these misconceptions' review. We began the paper by posing a problem: Can and should processes that cause scientific phenomena be categorized as either direct or emergent?, as suggested by the ontological shift theory. We showed that there are processes such as heat conduction and sound propagation, which under some conditions are direct, whereas under other conditions, they are emergent. Thus, we claimed that processes cannot be categorized as direct or emergent per se. We further showed that even if a process may be categorized as direct or emergent, more information about the phenomena can be gained if we view direct and emergent processes as edges of the same scale, which we called level of emergency. To do so, we connected between a system's order and direct or emergent concepts.

A system's order level deals with whether the system's elements have a distinct and predefined role, whether the interactions between the elements are predefined and whether they are sequential or parallel, whether the behavior of each element is predictable, and whether the system's behavior is predictable. In other words, a system's order relates to the quantity of information we have about the system. Analyzing the ontological shift theory, we showed that direct and emergent concepts deal with the same set of issues and thus may be described in terms of the system's order. In direct processes as opposed to emergent ones, the role of each element is distinct and predefined, and thus each element's behavior can be predicted, the interactions between the elements are predefined and occur sequentially, and there is a goal to be achieved. This means that direct processes are processes that have a high order, whereas emergent processes possess a low order.

We then suggested that direct and emergent processes should be viewed as extremes or limiting cases of the same scale that we called level of emergency. A low level of emergency means that the process is "more" direct, while a high level of order means that the process is "more" emergent. We then showed that entropy does exactly this: it measures the system's order level or the level of emergency. This means that low entropy indicates that the process is more direct while high entropy indicates that the process is more emergent. Finally, we provided examples of how the broadened shift theory, which now includes the idea of entropy, can be used in teaching about heat conduction and sound propagation. But these were only examples and we believe that the entropy idea can and should be implemented in other domains as well.

To help educators design learning environments that take into account the broadened ontological shift category theory that combines the entropy idea, we suggest that scientific process phenomena should be put on a level of emergency scale according to the following guidelines outlined in Table I.

Chi [1] argued that

“...when the to-be-learned concept is ontologically miscategorized [for instance, sound propagation is miscategorized as a direct process] then learning and instruction may require at least two additional processes. First, students have to be made aware that they need to shift their representation of the to-be-learned concept from one ontological structure to another. Second, the schema or structure to which they have to shift their representation, the emergent process 
TABLE I. Main guidelines for establishing the level of emergency of processes.

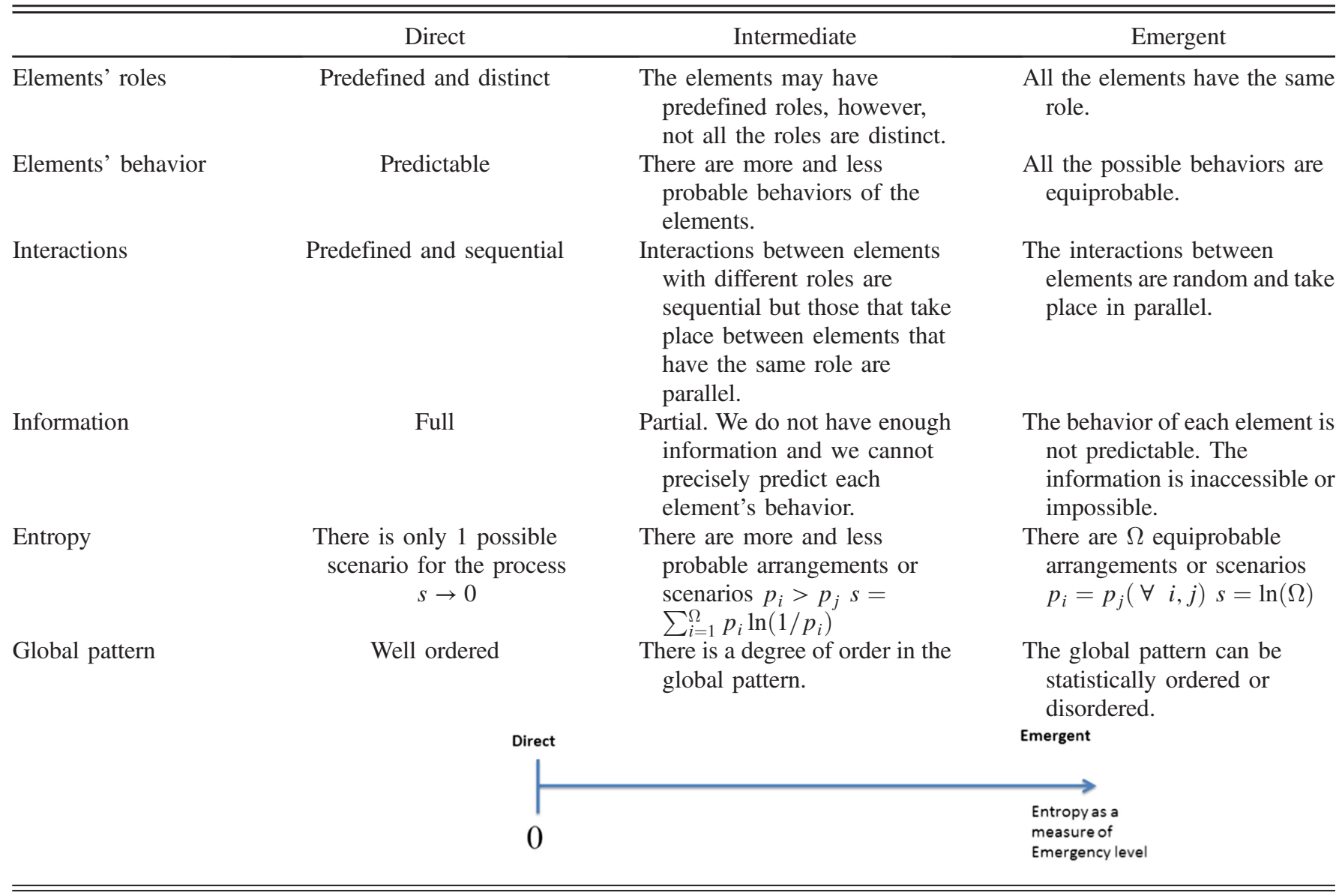

schema, may not already exist. This means that instruction has to focus on helping students build such a schema first." (p. 193).

Therefore, educators in such cases have to focus on teaching the underlying causal structure of emergent processes via the ontological attributes [1] (p. 194). Slotta and Chi [2] as well as Chi et al. [4] examined this claim of Chi empirically and found it to be valid. Interpreting scientific phenomena in terms of level of emergence, as we suggest here, is still based on the same idea of Chi, providing however, a more accurate way to analyze and understand physical processes and thus, as we believe, might promote students' understanding about the underlying mechanism explaining these phenomena. Indeed, if one goal of science education is to advance students' learning about their own world [21,40], then, making a "zoom-in" into the molecular or atomic patterns of statistical processes analyzing their ways of emergence should be an instrumental part of instruction. We believe that our refined version of Chi's framework might provide the students with a more detailed and accurate picture of the emergent processes' inner mechanisms, their laws, and regularities. Also, according to Chi $[1,2,4]$ educators should teach the general framework of level-of-emergence analysis on the first stage, applying simple examples such as the wolves' hunt. And only later, emergent physical phenomena should be introduced using the existing schema and being analyzed by the tools learned on the first stage.

Furthermore, we argued that such an interpretation enables comparing between different concepts, which might also promote an understanding of the concepts. Indeed, drawing comparisons of any kind is considered in the literature to require higher order thinking [41]. Indeed, "higher order thinking involves breaking down complex material into parts, detecting relationships, combining new and familiar information..." [41] (p. 21); all these are steps in the process of comparison by level of emergency. Drawing a comparison demands a deep understanding of each concept. The learner should also identify properties according to which the comparison should be conducted. It is known from the literature on analogical reasoning that while novices tend to draw comparisons based on nonrelevant physical properties, experts base their comparisons on physics principles [13]. Thus, if comparisons are based on the level of emergency or entropy, we 
can consider it as evidence for a deep expert understanding of the concepts. We illustrated this by comparing sound and heat. We believe that other comparisons of this kind should be introduced to the students. For instance, comparison between electric current vs heat conduction, or a particle's motion in Newtonian mechanics vs in quantum mechanics in terms of the level of emergency might promote an understanding of each of the concepts.
[1] M. T. H. Chi, Commonsense conceptions of emergent processes: Why some misconceptions are robust?, J. Learn. Sci. 14, 161 (2005).

[2] J. D. Slotta and M. T. H. Chi, Helping students understand challenging topics in science through ontology training, Cognit. Instr. 24, 261 (2006).

[3] M. T. H. Chi, Three types of conceptual change: Belief revision, mental model transformation, and categorical shift, Handbook of Research on Conceptual Change (Erlbaum, Hillsdale, NJ, 2008).

[4] M. T. H. Chi, R. D. Roscoe, J. D. Slotta, M. Roy, and C. C. Chase, Misconceived causal explanations for emergent processes, Cogn. Sci. 36, 1 (2012).

[5] M. T. H. Chi, Conceptual change within and across ontological categories: Examples from learning and discovery in science, Cognitive Models of Science: Minnesota Studies in the Philosophy of Science, edited by R. Giere (University of Minnesota Press, Minneapolis, 1992).

[6] H. Eshach and J. L. Schwartz, Sound stuff? Naive materialism in middle-school students' conceptions of sound, Int. J. Sci. Educ. 28, 733 (2006).

[7] M. C. Wittmann, Making sense of how students come to understanding of physics: An example of mechanical waves., Dissertation submitted in partial fulfillment of the requirements for the degree of Ph.D., Maryland University, United Kingdom, 1998.

[8] Z. Hrepic, Development of real-time assessment of students' mental models of sound propagation, Dissertation submitted in partial fulfillment of the requirements for the degree of Ph.D., Kansas State University, Manhattan, Kansas, 2004.

[9] Ch. Baily and D. Finkelstein, Development of quantum perspectives in modern physics, Phys. Rev. ST Phys. Educ. Res. 5, 010106 (2009).

[10] C. Periago, A. B. Pejuan, and B. Xavier, Misconceptions about the propagation of sound waves, Proceedings of the EAEEIE Annual Conference, (IEEE Xplore, Valencia, Spain, 2009).

[11] A. Volfson, Dialogue on sound waves: From particle to particle, Thesis submitted in partial fulfillment of the requirements for the degree of Master of Arts in the Faculty of Humanities and Social Sciences, Ben-Gurion University, 2011.

[12] M. Reiner, J. D. Slotta, M. T. H. Chi, and L. B. Resnick, Naïve physics reasoning: A commitment to substancebased conceptions, Cognit. Instr. 18, 1 (2000).

[13] M. T. H. Chi, P. J. Feltovich, and R. Glasser, Categorization and representation of physics problems by experts, and novices, Cogn. Sci. 5, 121 (1981).
[14] L. D. Landau and E. M. Lifshitz, Statistical Physics (Nauka, Moscow, 1964).

[15] I. V. Savel'ev, Course of General Physics, Molecular Physics and Thermodynamics, Vol. 3 (Nauka, Moscow, 1998).

[16] R. K. Pathria and P. D. Beale, Statistical Mechanics, 3rd ed. (Elsevier, Oxford, 2001).

[17] K. Huang, Statistical Mechanics, 2nd ed. (John Willey \& Sons, New York, 1987).

[18] Ch. Kittel and H. Kroemer, Thermal Physics, 2nd ed. (Freeman, New York, 1980).

[19] E. Keszei, Chemical Thermodynamics: An Introduction (Springer-Verlag, Berlin, 2012).

[20] D. L. Medin and L. J. Rips, Concepts and categories: Memory, meaning, and metaphysics, in The Cambridge Handbook of Thinking and Reasoning, edited by $\mathrm{K}$. Holyoak and B. Morrison (Cambridge University Press, Cambridge, England, 2005).

[21] H. Eshach, Development of a student-centered instrument to assess middle school students' conceptual understanding of sound, Phys. Rev. ST Phys. Educ. Res. 10, 010102 (2014).

[22] H. Eshach, T. Lin, and C. Tsai, Misconception of sound and conceptual change: A cross sectional study on students' materialistic thinking of sound, J. Res. Sci. Teach. 55, 664 (2018).

[23] S. V. Gromov, Physics 11, 4th ed. (Prosvescheniye, Moscow, 2003).

[24] J. S. Martin, N. A. Smith, and C. D. Francis, Removing the entropy from the definition of entropy: Clarifying the relationship between evolution, entropy, and the second law of thermodynamics, Evolution: Education and Outreach 6, 30 (2013).

[25] J. W. Clark, J. R. Thompson, and D. B. Mountcastle, Investigating student conceptual difficulties in thermodynamics across multiple disciplines: The first law and P-V diagrams, Proceedings of the 2014 ASEE Annual Conference \& Exposition, Indianapolis, Indiana (2014), https://peer.asee.org/20713.

[26] N. Mulop, K. Mohd Yusof, and Z. Tasir, A review on enhancing the teaching and learning of thermodynamics, Procedia-Social Behav. Sci. 56, 703 (2012).

[27] S. R. Pathare and H. C. Pradhan, Students' misconceptions about heat transfer mechanisms and elementary kinetic theory, Phys. Educ. 45, 629 (2010).

[28] J. Clement, The use of analogies and anchoring intuitions to remediate misconceptions in mechanics (ERIC Document Reproduction Service No. ED 291 604). 
[29] D. Halliday, R. Resnick, and J. Walker, Fundamentals of Physics, 10th ed. (Wiley, USA, 214).

[30] R. Hawkes, J. Iqbal, F. Mansour, M. Miler-Bolotin, and P. Williams, Physics for Scientists and Engineers: An Interactive Approach (Nelson Education Ltd., USA, 2014).

[31] E. M. Gershenson, N. N. Malov, and A. N. Mansurov, Molecular Physics (Academia, Moscow, 2000).

[32] E. A. Crone, C. Wendelken, L. van Leijenhorst, R. D. Honomichl, K. Christoff, and S. A. Bunge, Neurocognitive development of relational reasoning, Dev. Sci. 12, 55 (2009).

[33] L. Kotovsky and D. Gentner, Comparison and categorization in the development of relational similarity, Child Development 67, 2797 (1996).

[34] N. Podolefsky, The use of analogy in physics learning and instruction, Department of Physics, University Colorado, Comprehensive Exam paper (2005).
[35] M. T. Tomlinson and B. C. Love, When learning to classify by relations is easier than by features, Thinking Reasoning 16, 372 (2010).

[36] C. E. Shannon, A mathematical theory of communication, Bell Syst. Tech. J. 27, 379 (1948).

[37] D. R. Raichel, The Science and Applications of Acoustics (Springer New York, NY, 2006).

[38] J. K. Finn, Physical Chemistry in Depth (Springer-Verlag, Berlin, Heidelberg, 2009).

[39] R. P. Feynman, R. B. Leighton, and M. Sands, The Feynman Lectures on Physics, Book 4: Kinetics, Heat, Sound (Mir, Moscow, 1967).

[40] H. Eshach, Science Literacy in Primary Schools and Pre-Schools (Springer, Netherlands, 2006).

[41] F. J. King, L. Goodson, and F. Rohani, Higher order thinking skills: Definitions, strategies, assessment, Assessment and evaluation educational services program (1998), http://www .cala.fsu.edu/files/higher_order_thinking_skills.pdf. 